Review Article

\title{
Tongxinluo Capsule Combined with Atorvastatin for Coronary Heart Disease: A Systematic Review and Meta-Analysis
}

\author{
Qiao Liu $\left(\mathbb{D},{ }^{1}\right.$ Taiwei Dong, ${ }^{1}$ Miaomiao Xi $\left(\mathbb{D},{ }^{2}\right.$ Licheng Gou, ${ }^{3}$ Yang Bai, ${ }^{2}$ Lian Hou, ${ }^{2}$ Min Li, \\ Li Ou $\left(\mathbb{D},{ }^{1}\right.$ Feng Miao $\mathbb{D}^{2}{ }^{2}$ and Peifeng Wei $\mathbb{D}^{1,2}$ \\ ${ }^{1}$ Shaanxi University of Chinese Medicine, Xianyang 712046, China \\ ${ }^{2}$ The Second Affiliated Hospital of Shaanxi University of Chinese Medicine, Xianyang 712000, China \\ ${ }^{3}$ Shaanxi Provincial Hospital of Traditional Chinese Medicine, Xi'an 710000, China
}

Correspondence should be addressed to Feng Miao; 2936185813@qq.com and Peifeng Wei; weipeifeng@163.com

Received 20 April 2020; Revised 24 October 2020; Accepted 15 June 2021; Published 19 July 2021

Academic Editor: Im Hee Shin

Copyright $(2021$ Qiao Liu et al. This is an open access article distributed under the Creative Commons Attribution License, which permits unrestricted use, distribution, and reproduction in any medium, provided the original work is properly cited.

Introduction. Coronary heart disease (CHD) is a common clinical cardiovascular disease, and its morbidity and mortality rates are increasing, which brings a serious burden to the family and society. Dyslipidemia is one of the most important risk factors for CHD. However, it is difficult to reduce blood lipids to an ideal state with the administration of a statin alone. Tongxinluo capsule (TXLC), as a Chinese patent medicine, has received extensive attention in the treatment of CHD in recent years. This systematic review and meta-analysis aim to provide evidence-based medicine for TXLC combined with atorvastatin in the treatment of CHD. Objective. To evaluate systematically the effectiveness and safety of TXLC combined with atorvastatin in the treatment of CHD. Methods. Seven English and Chinese electronic databases (PubMed, Cochrane Library, Embase, CNKI, VIP, CBM, and Wanfang) were searched from inception to January 2020, to search for randomized controlled trials (RCTs) on TXLC combined with atorvastatin in the treatment of CHD. Two researchers independently screened the literature according to the literature inclusion and exclusion criteria and performed quality assessment and data extraction on the included RCTs. We performed a systematic review following Cochrane Collaboration Handbook and Preferred Reporting Items for Systematic Reviews and Meta-Analyses (PRISMA) guidelines and using a measurement tool to assess the methodological quality of systematic reviews (AMSTAR 2). The quality of outcomes was evaluated by the Grading of Recommendations Assessment, Development and Evaluation (GRADE). And meta-analysis was performed by Review Manager 5.2. Results. A total of 15 RCTs with 1,578 participants were included in this review. Compared to atorvastatin treatment, TXLC combined with atorvastatin treatment showed potent efficacy when it came to the effectiveness of clinical treatment $(\mathrm{RR}=1.24 ; 95 \% \mathrm{CI}, 1.18,1.29 ; P<0.00001)$, total cholesterol $(\mathrm{TC}$; $\mathrm{MD}=-1.21 ; 95 \% \mathrm{CI}$, $-1.53,-0.89 ; P<0.00001$ ), triacylglycerol (TG; $\mathrm{MD}=-0.73 ; 95 \% \mathrm{CI},-0.81,-0.65 ; P<0.00001$ ), high-density lipoprotein cholesterol (HDL-C; MD = 0.27; 95\% CI, 0.23, 0.31; $P<0.00001$ ), low-density lipoprotein cholesterol (LDL-C; MD = -0.72; 95\% $\mathrm{CI},-0.80,-0.64 ; P<0.00001)$, C-reactive protein (CRP; SMD $=-2.06 ; 95 \% \mathrm{CI},-2.56,-1.57 ; P<0.00001$ ), frequency of angina pectoris $(\mathrm{SMD}=-1.41 ; 95 \% \mathrm{CI},-1.97,-0.85 ; P<0.00001)$, duration of angina pectoris $(\mathrm{MD}=-2.30 ; 95 \% \mathrm{CI},-3.39,-1.21$; $P<0.0001)$, and adverse reactions $(\mathrm{RR}=0.84 ; 95 \% \mathrm{CI}, 0.51,1.39 ; P=0.50)$. No serious adverse events or reactions were mentioned in these RCTs. According to the PRISMA guidelines, although all studies were not fully reported in accordance with the checklist item, the reported items exceeded $80 \%$ of all items. With the AMSTAR 2 standard, the methodological quality assessment found that 9 studies were rated low quality and 6 studies were rated critically low quality. Based on the results of the systematic review, the GRADE system recommended ranking method was used to evaluate the quality of evidence and the recommendation level. The results showed that the level of evidence was low, and the recommendation intensity was a weak recommendation. Conclusions. TXLC combined with atorvastatin in the treatment of CHD can effectively improve the effectiveness of clinical treatment, significantly reduce the frequency and duration of angina pectoris, decrease blood lipids, and improve inflammatory factors. However, due to the low quality of the literature included in these studies and the variability of the evaluation methods of each study, there is still a need for a more high-quality, large sample, multicenter clinical randomized control for further demonstration. 


\section{Introduction}

Coronary atherosclerotic heart disease, referred to as coronary heart disease (CHD), is caused by coronary atherosclerosis or spasm that causes the lumen to become blocked and narrow, blood flow to be blocked, and blood supply to become insufficient, which causes myocardial ischemia and no oxygen supply. A cardiovascular disease that causes necrosis is one of the diseases with the highest morbidity and mortality in the world $[1,2]$. A large number of clinical studies have shown that the number of patients with CHD is increasing, and due to bad lifestyle habits such as smoking and drinking, the incidence trend is gradually showing younger age [3]. Patients mainly show symptoms such as angina pectoris and heart failure during attacks. In severe cases, they may even die suddenly [4], which greatly affects the daily life of patients and even threatens their lives. Therefore, safe and effective treatments are needed to prevent and treat this disease.

According to research, the incidence of cardiovascular disease can be reflected by blood lipid levels [5]. In patients with a high risk of $\mathrm{CHD}$ and similar critical conditions, low-density lipoprotein cholesterol (LDL-C) should be reduced by at least $30-50 \%$ in order to obtain a clinical benefit [6]. So the treatment of $\mathrm{CHD}$ can improve blood lipid levels by lipidlowering drugs, effectively reduce LDL-C levels, and prevent the formation or development of coronary atherosclerotic plaque, thereby preventing the plaque from further rupture and thrombosis that may lead to myocardial infarction. Atorvastatin is commonly used clinically to treat CHD. It is a reductase inhibitor that can inhibit the synthesis of cholesterol, reduce TG levels, increase the activity of LDL receptors, and promote the metabolism of LDL-C [7]. The application of statins significantly reduced the incidence of adverse cardiovascular events and delayed the progression of coronary atherosclerosis [8]. However, it has been found in clinical practice that some patients with CHD still have adverse cardiovascular events after treatment with strong statins $[9,10]$. CHD belongs to the category of chest obstruction, palpitations, and heartache in traditional Chinese medicine (TCM), and the clinical practice of Chinese medicine has confirmed that blood stasis is the core pathogenesis of chest obstruction [11]. Studies have shown that on the basis of statin therapy, the addition of TCM for promoting blood circulation and removing blood stasis can improve the efficacy of CHD [12]. Tongxinluo capsule (TXLC) is a kind of TCM preparation, mainly composed of ginseng, leech, whole scorpion, centipede, chuanxiong, and borneol, which has the effects of promoting blood circulation and removing blood stasis [13]. Modern pharmacological studies have shown that TXLC can lower cholesterol, improve microcirculation, relieve atherosclerosis, stabilize plaque, and inhibit inflammatory cell infiltration [14]. In recent years, it is used to treat CHD and has achieved good results. This study will perform a meta-analysis of the randomized controlled trials (RCTs) of TXLC combined with atorvastatin in the treatment of $\mathrm{CHD}$ and appropriately refers to relevant literature methods [15], in order to evaluate the efficacy and safety of the two drugs in the treatment of CHD.

\section{Methods}

2.1. Search Strategy. Comprehensive searches were conducted in both English and Chinese databases to identify all published RCTs from inception to January 2020. All relevant RCTs were searched from the following 7 databases including PubMed, Cochrane Library, Embase, CNKI, VIP, CBM, and Wanfang. The following search terms were used: "tongxinluo capsules" (title/abstract), AND "atorvastatin" (title/abstract), AND "cardiovascular disease" (title/abstract), OR "coronary heart disease" (title/abstract), OR "coronary artery disease" (title/abstract), OR "acute coronary syndrome" (title/abstract).

2.2. Inclusion Criteria. Two authors (Qiao Liu and Taiwei Dong) read the titles and abstracts of trials in all searched databases independently to assess the rationality for inclusion. The full text was further read to evaluate for the inclusion criteria. The inclusion criteria were as follows. (1) Diagnostic criteria for CHD: 1979 "Nomenclature and diagnostic criteria for ischemic heart disease" developed by World Health Organization (WHO). (2) Research protocol: the treatment group received TXLC and atorvastatin treatment, and the control group received atorvastatin or conventional and atorvastatin treatments. (3) Outcome indicators: the effectiveness of clinical treatment is the proportion of the total number of people who are significantly effective and effective. According to the WHO standard of CHD curative effect, it is divided as follows: significantly effective: angina pectoris symptoms disappeared significantly, and the electrocardiogram returned to normal; effective: angina pectoris symptoms were reduced to a certain extent, and the electrocardiogram was improved; and invalid: angina pectoris symptoms did not change, and the electrocardiogram did not change. Blood lipid levels include total cholesterol (TC), triglycerides (TG), low-degree lipoprotein cholesterol (LDL-C), high-density lipoprotein cholesterol (HDL-C), inflammatory factors including C-reactive protein (CRP), and adverse reactions. (4) Research type: randomized controlled trials (RCTs).

2.3. Exclusion Criteria. The trials conforming to the following conditions were excluded: (1) noncardiogenic chest pain; (2) cases included in the study that included myocardial infarction, rheumatic heart disease, cardiomyopathy, severe heart rhythm disorders, severe heart failure, and so on; (3) reduplicative publications reporting the same trials; (4) nonrandomized controlled trials; (5) nonclinical experiments, reviews, literature research, mechanism research, or animal experiment; (6) controlled interventions combined with any other medicine in the control or treatment group; (7) incorrect data for meta-analysis; (8) patients with unclear functional classification; and (9) trials with unclear evaluation indicators or basic data for statistic research. 
2.4. Reporting Quality of Included RCTs. The Preferred Reporting Items for Systematic Reviews and Meta-Analyses (PRISMA) statement [16] is composed of a 27-item checklist and a 4-phase flow diagram. The 7 parts of the checklist item are the title, abstract, introduction, methods, results, discussion, and funding. Each item has options such as "yes," "no," or "not applicable". The flow diagram is composed of identification, screening, eligibility, and included.

2.5. Data Extraction. Based on the search strategy, two investigators (Taiwei Dong and Miaomiao Xi) combined the literature inclusion and exclusion criteria, independently screened the literature, excluded the irrelevant literature, and checked it. When there was a disagreement, the third researcher (Feng Miao) participated in the discussion and jointly evaluated the basic content including the first author and publication year; random method; number of cases in the treatment and control groups; age; gender; specific intervention measures and course of treatment; and outcome indicators.

\subsection{Evaluation of Literature Quality}

2.6.1. Assessment of Risk of Bias. Two researchers (Qiao Liu and Taiwei Dong) independently assessed the included RCTs based on the bias risk assessment criteria recommended by the Cochrane Collaboration Handbook. The methodological criteria and methods of evaluation are as follows: (1) random sequence generation, (2) allocation concealment, (3) blinding of participants and personnel, (4) blinding of outcome assessment, (5) incomplete outcome data, (6) selective reporting, and (7) other bias. We conduct bias risk assessments for each RCT and classify them as "high risk," "uncertain risk," or "low risk." Two researchers discussed according to the above criteria and methods, and if necessary, they could intervene through a third evaluator (Peifeng Wei) to negotiate and finally reach a consensus.

2.6.2. Quality Assessment of Systematic Reviews. The methodological quality of all included RCTs was assessed using Assessing the Methodological Quality of Systematic Reviews (AMSTAR 2) [17]. The domain-specific questions in AMSTAR 2 are framed so that a "yes" answer denotes a positive result. If no information is provided to rate an item, the item should be rated as a "no." We can choose a "partial yes" response in some instances where we considered it worthwhile to identify partial adherence to the standard. A detailed description of AMSTAR 2 is provided in Table 1 . Based on the information provided by each RCT, two researchers conducted methodological quality assessments through AMSTAR 2. If there is a disagreement, the third researcher can negotiate a settlement.

2.6.3. Evidence Quality and Recommendation Level. Based on the results of systematic reviews, the GRADE system recommended ranking method [33] was used to evaluate the quality of evidence and the recommendation level. GRADE evidence quality assessment can divide the importance of the assessment results into 3 levels, of which
1-3 are unimportant outcome indicators, 4-6 are important outcome indicators, and 7-9 are critical outcome indicators. Since the treatment of CHD is mainly to improve the patient's condition by reducing blood lipids, 5 indicators of clinical treatment effectiveness, TC, TG, HDL-C, and LDL-C are used as critical outcome indicators. CRP, frequency of angina pectoris, duration of angina pectoris, and adverse reactions were used as important outcome indicators. RCTs were initially defined as high-quality evidence, and observational studies are defined as low-quality evidence. According to the research design, the further evidence on increase and decrease factors are determined by 5 downgrade factors and 4 upgrade factors [34]. The recommendation level is divided into "strong recommendation" and "weak recommendation": strong recommendation indicates that the evaluator is convinced that the intervention has more advantages than disadvantages or disadvantages than benefits, and weak recommendation indicates that the advantages and disadvantages are uncertain. Finally, GRADEpro 3.6 software was used to analyze and chart the quality of evidence, and the recommended level was given based on the quality of evidence combined with the research theme. The evaluation of the promotion and demotion factors is the responsibility of QiaoLiu and Miaomiao Xi. If there is a dispute, the third researcher (Peifeng Wei) is required to review and reach an agreement through discussion.

2.7. Statistical Analysis of Data. Reviewer Manager 5.2 software provided by Cochrane was used for meta-analysis of the literature. For outcome measures, dichotomous variables were presented as risk ratio (RR) with 95\% confidence intervals (CI), while continuous outcomes were expressed as mean difference (MD) with 95\% CI; if each trial data uses different measurement tools and different measurement units to record data, the standardized mean difference (SMD) is used for analysis. As a quantitative measure of inconsistency, the $I$-square $\left(I^{2}\right)$ statistic was used to assess heterogeneity. The fixed effects model was performed with minor heterogeneity when $I^{2}$ was less than $50 \%$. The random effects model was applied when $I^{2}$ was over $50 \%$. Meanwhile, a funnel plot was used for assessing the potential publication bias. The data was entered by Qiao Liu and supervised by Taiwei Dong.

\section{Results}

3.1. Literature Screening Process and Results. The PRISMA flow diagram is presented in Figure 1. A total of 182 records were identified for preliminary screening after searching English and Chinese databases. All the included trials were conducted in China and published in Chinese. 99 records were reserved for further screening after removing $83 \mathrm{du}-$ plicated publications. For the preserved records, 62 obvious irrelevant literature were excluded by reading the title and abstract. 37 full-text articles were used for further assessment. After reading the full text, 22 more literature works were excluded for the following reasons: participants not meeting the inclusion criteria $(n=3)$; improper grouping, 
TABLE 1: AMSTAR 2 assessment for included meta-analyses.

\begin{tabular}{|c|c|c|c|c|c|c|c|c|c|c|c|c|c|c|c|c|c|}
\hline \multirow{2}{*}{ Authors and year of included studies } & \multicolumn{17}{|c|}{ AMSTAR 2 item } \\
\hline & 1 & $2^{*}$ & 3 & $4^{*}$ & 5 & 6 & $7^{*}$ & 8 & $9^{*}$ & 10 & $11^{*}$ & 12 & $13^{*}$ & 14 & $15^{*}$ & 16 & Ranking of quality \\
\hline Hang et al. 2016 [18] & Yes & No & Yes & PY & Yes & Yes & PY & PY & PY & No & Yes & Yes & Yes & Yes & Yes & Yes & Low \\
\hline Liu and An 2016 [19] & Yes & No & Yes & PY & Yes & Yes & PY & PY & PY & No & No & Yes & Yes & No & Yes & Yes & Critically low \\
\hline Qiao $2017[20]$ & Yes & No & Yes & PY & Yes & Yes & PY & PY & PY & No & No & Yes & Yes & Yes & Yes & Yes & Critically low \\
\hline Song 2018 [21] & Yes & No & Yes & PY & Yes & Yes & PY & PY & PY & No & No & Yes & Yes & No & No & Yes & Critically low \\
\hline Nie 2018 [22] & Yes & No & Yes & PY & Yes & Yes & PY & PY & PY & No & Yes & Yes & Yes & Yes & Yes & Yes & Low \\
\hline Li 2017 [23] & Yes & No & Yes & PY & Yes & Yes & PY & PY & PY & No & Yes & Yes & Yes & Yes & Yes & Yes & Low \\
\hline 2018 & Yes & No & Yes & PY & Yes & Yes & PY & PY & PY & No & Yes & Yes & Yes & Yes & Yes & Yes & Lo \\
\hline Zhac & Yes & No & Yes & PY & Yes & Yes & PY & PY & PY & No & Yes & Yes & Yes & Yes & Yes & Yes & \\
\hline Qian 2014 [26] & Yes & No & Yes & PY & Yes & Yes & PY & PY & PY & No & Yes & Yes & Yes & Yes & Yes & Yes & Low \\
\hline Qiao 2017 [27] & Yes & No & Yes & PY & Yes & Yes & PY & PY & PY & No & Yes & Yes & Yes & Yes & Yes & Yes & Low \\
\hline Jiang et al. 2019 [28] & Yes & No & Yes & PY & Yes & Yes & PY & PY & PY & No & Yes & Yes & Yes & Yes & Yes & Yes & Lo \\
\hline & Yes & No & Yes & PY & Yes & Yes & PY & PY & PY & No & No & No & No & No & Yes & Yes & Critically low \\
\hline Wang 2018 [30] & Yes & No & Yes & PY & Yes & Yes & PY & PY & PY & No & Yes & Yes & Yes & Yes & Yes & Yes & Low \\
\hline and Ding 2012 [31] & Yes & No & Yes & PY & Yes & Yes & PY & PY & PY & No & No & Yes & Yes & No & Yes & Yes & Critically low \\
\hline Zeng 2012 [32] & Yes & No & Yes & PY & Yes & Yes & PY & PY & PY & No & No & Yes & Yes & No & Yes & Yes & Critically low \\
\hline No. of yes & 15 & 0 & 15 & 0 & 15 & 15 & 0 & 0 & 0 & 0 & 9 & 14 & 14 & 10 & 14 & 15 & \\
\hline
\end{tabular}

*The critical items of the AMSTAR 2; PY: partial yes; high: no or one noncritical weakness; moderate: more than one noncritical weakness; low: one critical flaw with or without noncritical weaknesses; and critically low: more than one critical flaw with or without noncritical weaknesses.

outcomes, or pharmacy $(n=10)$; nonrandomized controlled trials $(n=5)$; and no data available for extraction $(n=4)$. Finally, 15 RCTs of TXLC combined with atorvastatin for $\mathrm{CHD}$ were included in this review.

3.2. Study Characteristics. As shown in Table 2, a total of 15 RCTs with 1,578 participants were included in this review. The control group consisted of 789 patients, while the treatment group consisted of 789 patients. All trials' sample sizes ranged from 60 to 160 . As for the characteristics of the intervention, the course of treatment varied from 4 weeks to 3 months. Only 1 trial did not mention the course of treatment [20]. The baseline of patients in both groups was balanced. The treatment group used TXLC combined with the same atorvastatin and conventional treatment as a control group, and 4 trials used only atorvastatin $[18,20,21,24]$. Most trials in the treatment group used the dose of 12 capsules per day; 3 trials used 9 capsules per day; and only 1 trial used 6 to 12 capsules per day. TXLC was given through oral administration 3 times daily in all included trials. Most trials in the control group used atorvastatin dose of $20 \mathrm{mg} / \mathrm{d} ; 2$ trials used $10 \mathrm{mg} / \mathrm{d} ; 2$ trials used 1 tablet per day; and only 1 trial adjusted the dosage according to the specific conditions of the patient. The control group used conventional medical treatment, including nitrate drugs, aspirin antiplatelet therapy, heparin anticoagulant therapy, and $\beta$-receptor blockers. Fourteen trials reported the effectiveness of clinical treatment. Nine trials reported TC and TG. Eight trials reported LDL-C. Seven articles reported CRP. Six articles reported HDL-C and adverse reactions. Five trials reported the frequency of angina pectoris. Four trials reported the duration of angina pectoris.

3.3. Reporting Quality Results of Included RCTs. The included RCTs were not well reported due to the incomplete implementation of the PRISMA statement. None of the studies reported on protocol and registration; to avoid or minimize the risk of bias in a study [29], six studies [19-21, 29, 31, 32] were not subjected to additional analysis, and the remaining projects were fully reported. PRISMA's checklist item is shown in Table 3.

3.4. Quality Assessment of Systematic Reviews. According to the AMSTAR 2 standard for methodological quality evaluation, 9 studies were rated as "low", and 6 studies were rated as "critically low." None of the studies mentioned that the review method was established before the review was conducted, and none of the studies reported the source of funding; 6 studies [19-21, 29, 31, 32] failed to combine the results statistically; 1 study [29] did not assess the risk of bias; 4 studies [19, 21, 31, 32] failed to provide a satisfactory explanation for the existence of heterogeneity; and 1 study [21] failed to adequately investigate publication bias (Table 1).

3.5. Risk of Bias Assessment in Included RCTs. Six of the included RCTs $[18,21,23,25,27,30]$ used the random number table method for allocation, and the remaining five trials $[19,20,22,24,26]$ only mentioned random allocation, but there is no specific description of the random method, Only one reported that random sequence was parity of hospital order [29]. All trials do not mention whether to use allocation hiding and whether to blind doctors and patients. No subjects who dropped out. And other potential sources of bias are unclear as shown in Figure 2.

AMSTAR 2 checklist is as follows:

(1) Did the research questions and inclusion criteria for the review include the components of PICO?

(2) Did the report of the review contain an explicit statement that the review methods were established 


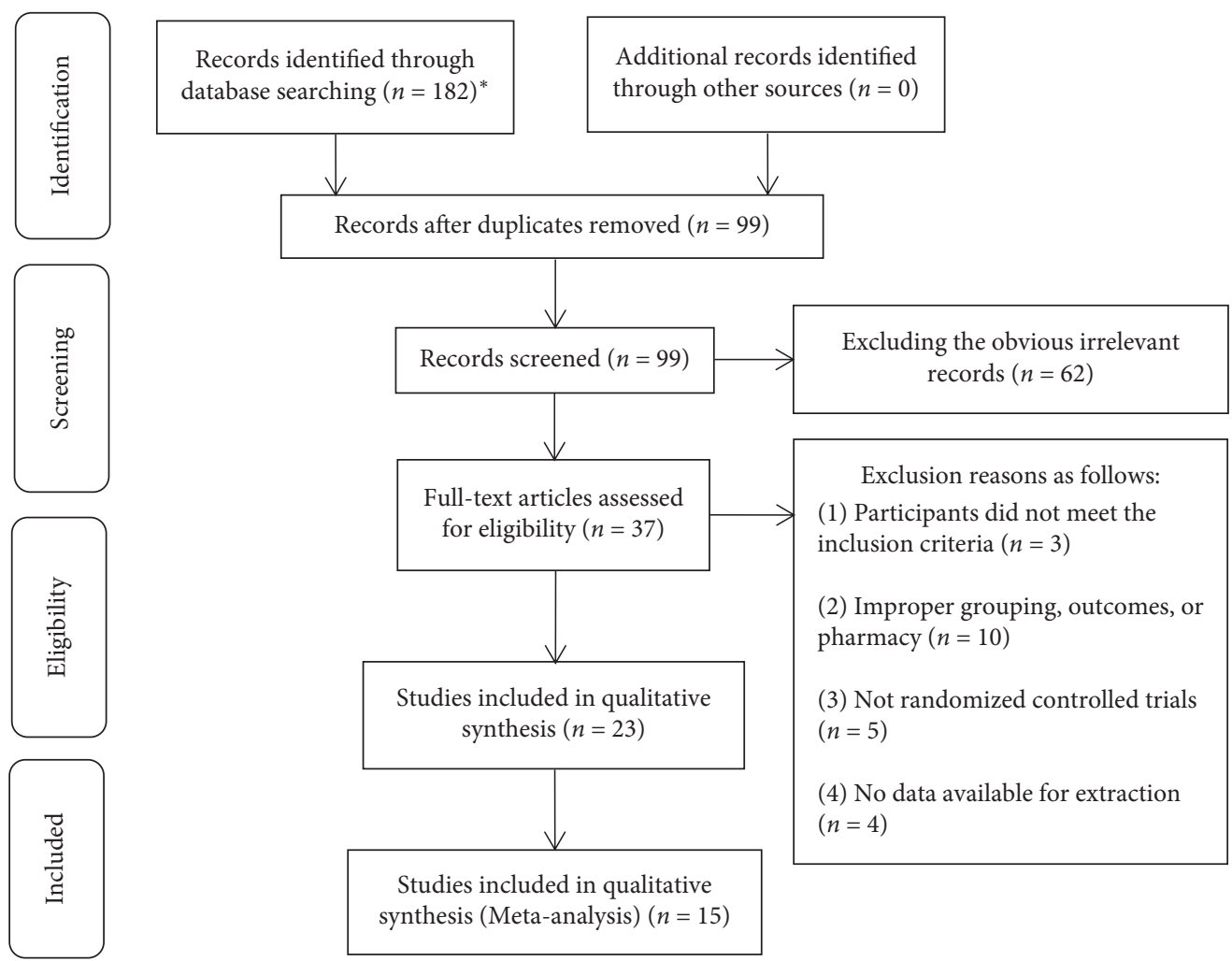

Figure 1: Literature search: PRISMA flow diagram. ${ }^{*}$ The number of databases and documents retrieved are as follows: PubMed $(n=3)$, Cochrane Library $(n=0)$, Embase $(n=0)$, CNKI $(n=54)$, VIP $(n=39)$, CBM $(n=27)$, and Wanfang $(n=59)$.

prior to the conduct of the review, and did the report justify any significant deviations from the protocol?

(3) Did the review authors explain their selection of the study designs for inclusion in the review?

(4) Did the review authors use a comprehensive literature search strategy?

(5) Did the review authors perform study selection in duplicate?

(6) Did the review authors perform data extraction in duplicate?

(7) Did the review authors provide a list of excluded studies and justify the exclusions?

(8) Did the review authors describe the included studies in adequate detail?

(9) Did the review authors use a satisfactory technique for assessing the risk of bias (RoB) in individual studies that were included in the review?

(10) Did the review authors report on the sources of funding for the studies included in the review?

(11) If meta-analysis was performed, did the review authors use appropriate methods for statistical combination of results?

(12) If meta-analysis was performed, did the review authors assess the potential impact of RoB in individual studies on the results of the meta-analysis or other evidence synthesis?
(13) Did the review authors account for RoB in individual studies when interpreting/discussing the results of the review?

(14) Did the review authors provide a satisfactory explanation for, and discussion of, any heterogeneity observed in the results of the review?

(15) If they performed quantitative synthesis, did the review authors carry out an adequate investigation of publication bias (small study bias) and discuss its likely impact on the results of the review?

(16) Did the review authors report any potential sources of conflict of interest, including any funding they received for conducting the review?

\subsection{Meta-Analysis Results}

3.6.1. Meta-Analysis Based on the Effectiveness of Clinical Treatment. A total of 14 trials with 1,480 patients investigated the effectiveness of clinical treatment of TXLC plus atorvastatin versus atorvastatin in patients with $\mathrm{CHD}$ $[18-29,31,32]$. There were 740 patients in the treatment group and 740 in the control group. The results showed that there was no heterogeneity $\left(P=0.85 ; I^{2}=0 \%\right)$, and the fixed effects model was adopted for analysis. As shown in the forest plot, there was a statistically significant difference between TXLC plus atorvastatin and atorvastatin in the effectiveness of clinical treatment $(\mathrm{RR}=1.24$; 95\% CI, 1.18 , 1.29; $P<0.00001$; Figure 3). 


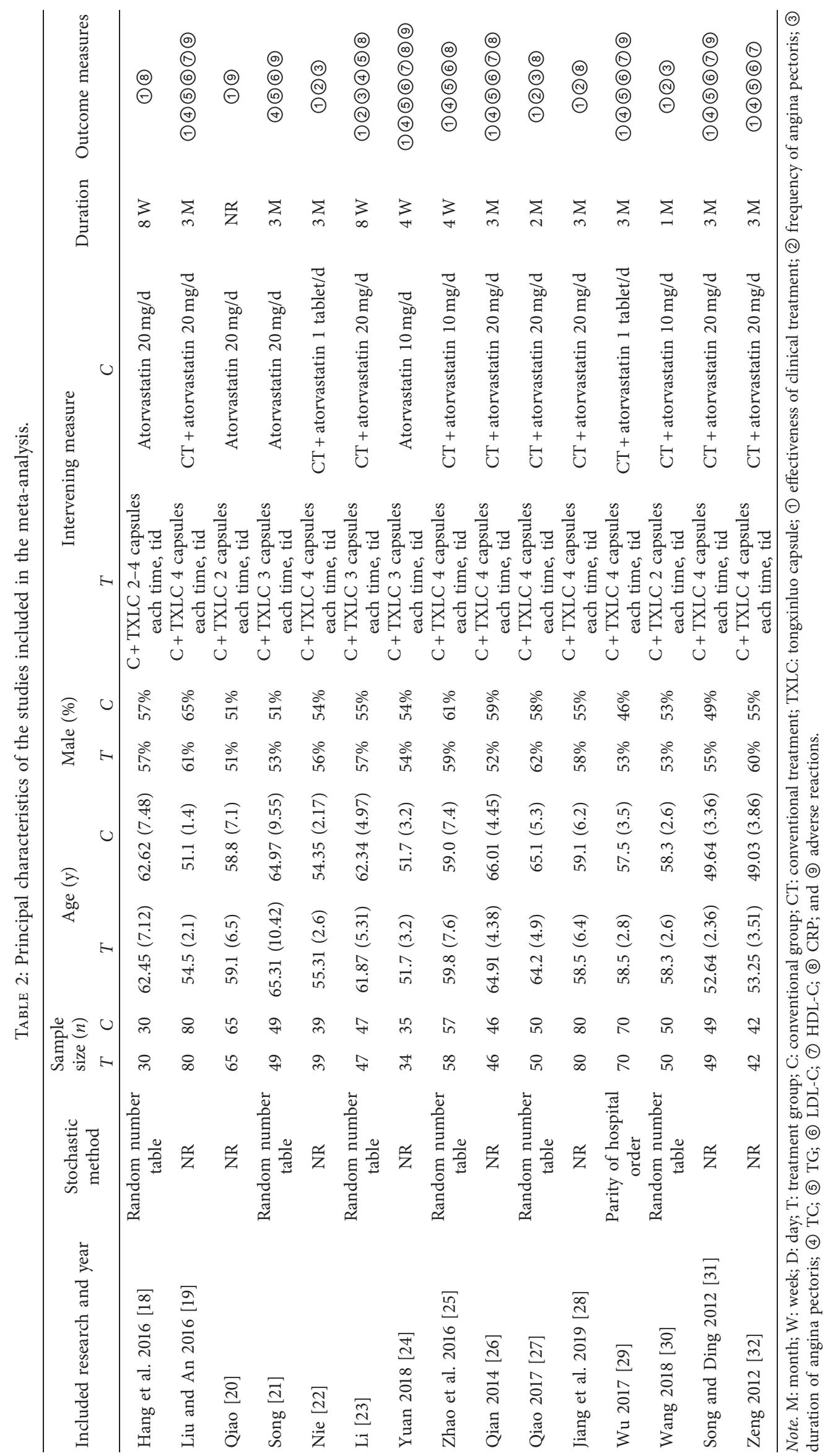




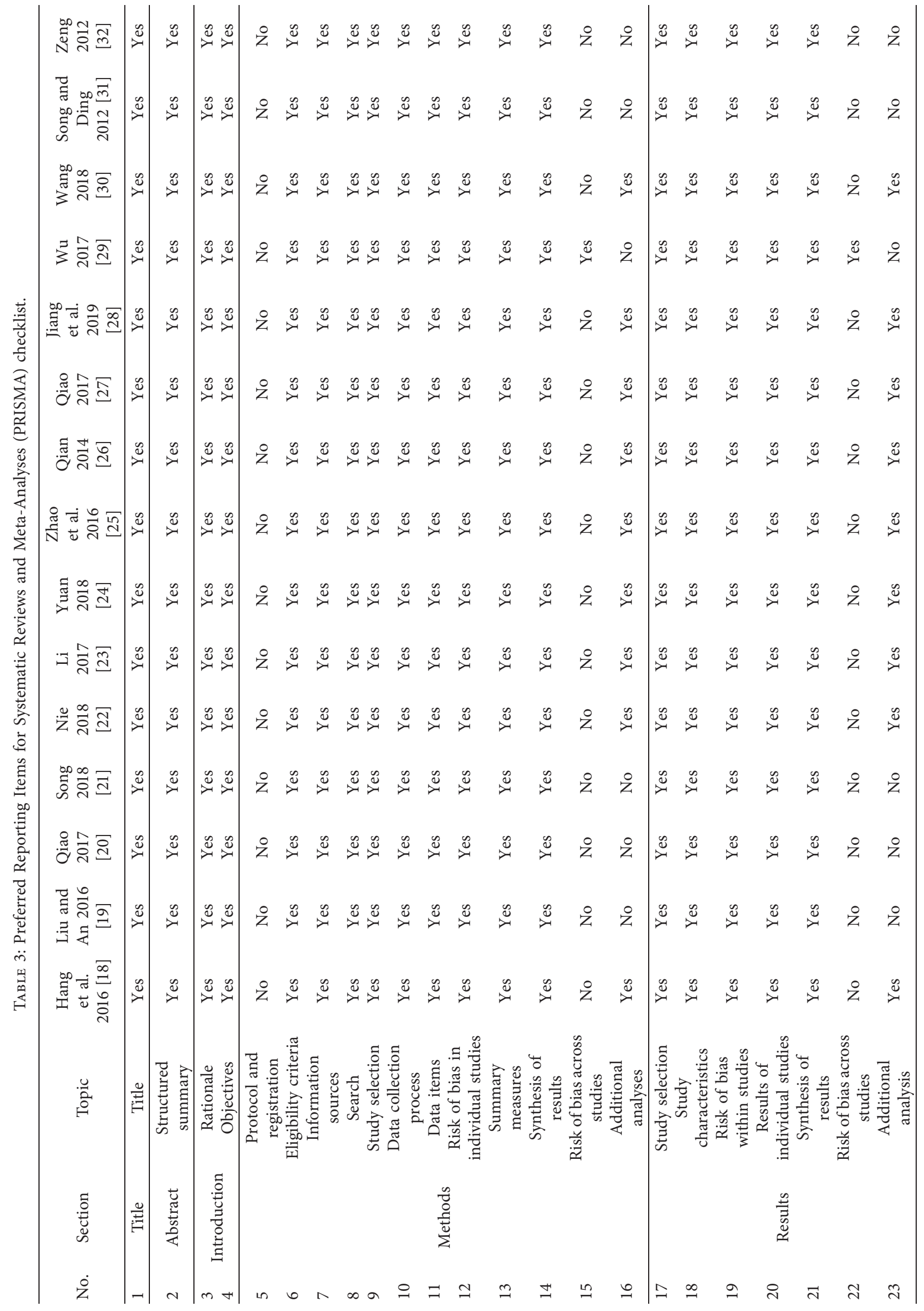




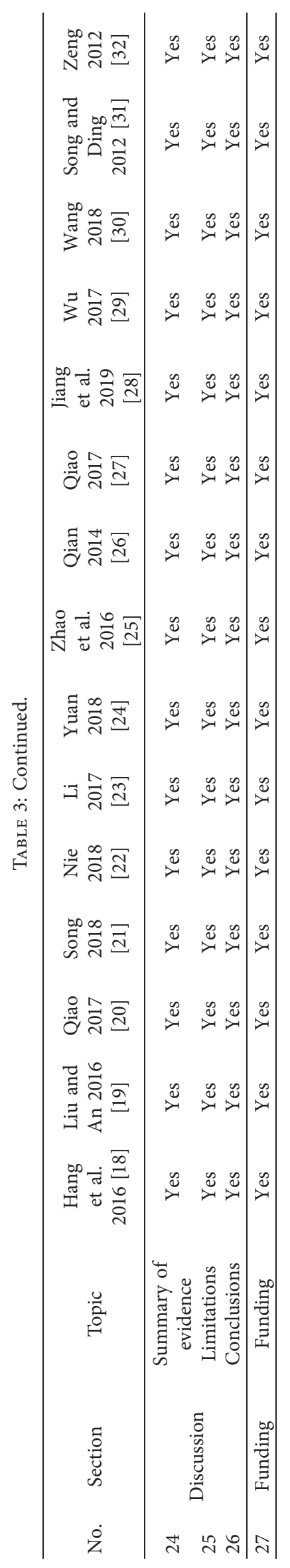




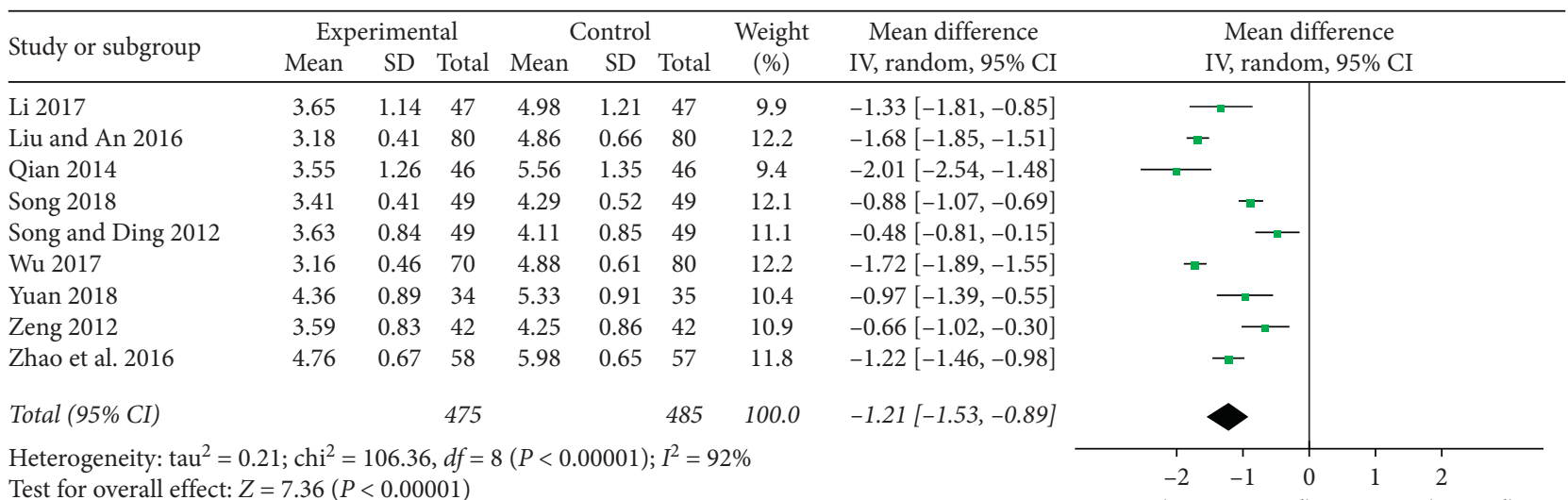

Test for overall effect: $Z=7.36(P<0.00001)$

Favours (experimental) Favours (control)

(a)

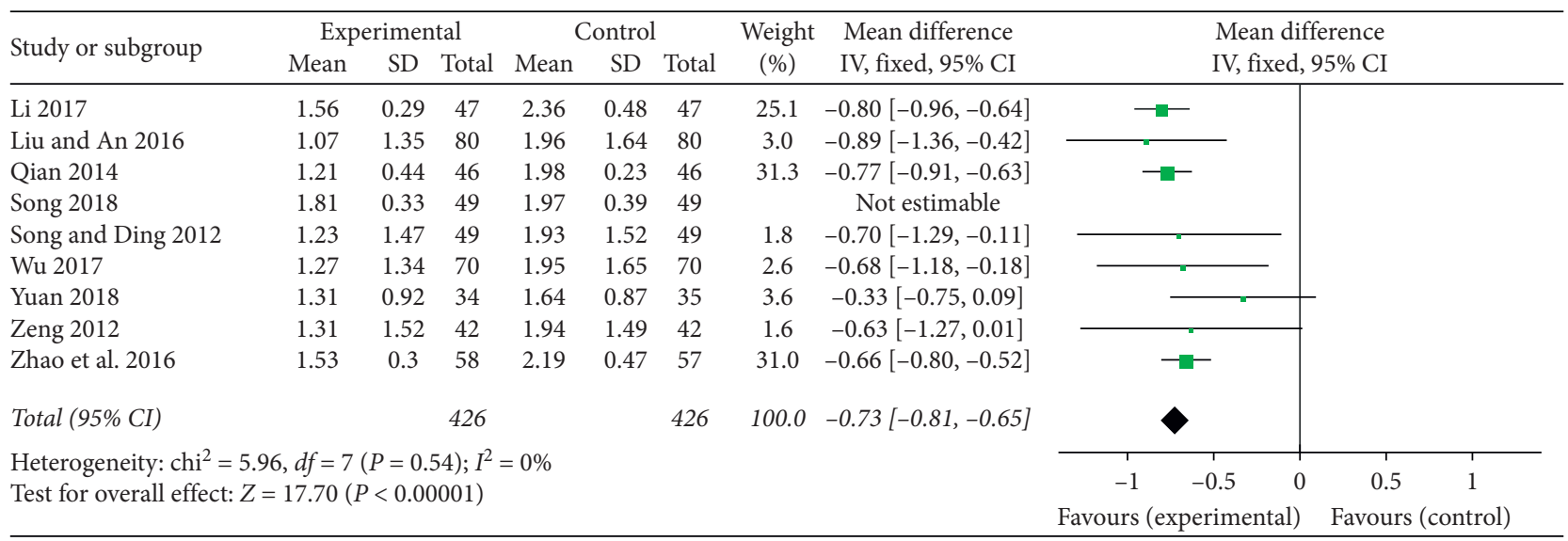

(b)

\begin{tabular}{|c|c|c|c|c|c|c|c|c|c|c|c|c|c|}
\hline \multirow{3}{*}{$\begin{array}{l}\text { Study or subgroup } \\
\text { Liu and An } 2016\end{array}$} & \multicolumn{3}{|c|}{ Experimental } & \multicolumn{3}{|c|}{ Control } & \multirow{2}{*}{$\begin{array}{l}\text { Weight } \\
(\%)\end{array}$} & \multirow{2}{*}{$\begin{array}{l}\text { Mean difference } \\
\text { IV, fixed, 95\% CI }\end{array}$} & \multirow{2}{*}{\multicolumn{5}{|c|}{$\begin{array}{l}\text { Mean difference } \\
\text { IV, fixed, } 95 \% \text { CI }\end{array}$}} \\
\hline & Mean & $\mathrm{SD}$ & Total & Mean & $\mathrm{SD}$ & Total & & & & & & & \\
\hline & 1.66 & 0.25 & 80 & 1.37 & 0.19 & 80 & 36.3 & $0.29[0.22,0.36]$ & & & & - & \\
\hline Qian 2014 & 1.41 & 0.38 & 46 & 1.11 & 0.29 & 46 & 9.0 & $0.30[0.16,0.44]$ & & & & & \\
\hline Song and Ding 2012 & 1.32 & 0.28 & 49 & 1.13 & 0.36 & 49 & 10.5 & $0.19[0.06,0.32]$ & & & & - & \\
\hline Wu 2017 & 1.67 & 0.29 & 70 & 1.36 & 0.15 & 70 & 29.4 & $0.31[0.23,0.39]$ & & & & 一 & \\
\hline Yuan 2018 & 1.37 & 0.29 & 34 & 1.18 & 0.44 & 35 & 5.6 & $0.19[0.01,0.37]$ & & & & & \\
\hline Zeng 2012 & 1.3 & 0.29 & 42 & 1.15 & 0.35 & 42 & 9.1 & $0.15[0.01,0.29]$ & & & & & \\
\hline Total (95\% CI) & & & 321 & & & 322 & 100.0 & $0.27[0.23,0.31]$ & & & & & \\
\hline \multirow{2}{*}{\multicolumn{9}{|c|}{$\begin{array}{l}\text { Heterogeneity: } \mathrm{Chi}^{2}=6.78, d f=5(P=0.24) ; I^{2}=26 \% \\
\text { Test for overall effect: } Z=12.66(P<0.00001)\end{array}$}} & -0.5 & -0.25 & 0 & 0.25 & 0.5 \\
\hline & & & & & & & & & & urs (control) & & $(\exp$ & ental) \\
\hline
\end{tabular}

(c)

\begin{tabular}{|c|c|c|c|c|c|c|c|c|c|c|}
\hline \multirow{3}{*}{$\begin{array}{l}\text { Study or subgroup } \\
\text { Liu and An } 2016\end{array}$} & \multicolumn{3}{|c|}{ Experimental } & \multicolumn{3}{|c|}{ Control } & \multirow{3}{*}{$\begin{array}{c}\text { Weight } \\
(\%)\end{array}$} & \multirow{3}{*}{$\begin{array}{c}\text { Mean difference } \\
\text { IV, fixed, 95\% CI } \\
-0.75[-1.00,-0.50]\end{array}$} & \multirow{2}{*}{\multicolumn{2}{|c|}{$\begin{array}{l}\text { Mean difference } \\
\text { IV, fixed, } 95 \% \text { CI }\end{array}$}} \\
\hline & \multirow{2}{*}{$\frac{\text { Mean }}{1.36}$} & \multirow{2}{*}{$\frac{\mathrm{SD}}{0.68}$} & \multirow{2}{*}{$\frac{\text { Total }}{80}$} & \multirow{2}{*}{$\frac{\text { Mean }}{2.11}$} & \multirow{2}{*}{$\begin{array}{l}\text { SD } \\
0.9\end{array}$} & \multirow{2}{*}{$\frac{\text { Total }}{80}$} & & & & \\
\hline & & & & & & & & & $\square$ & \\
\hline Qian 2014 & 1.66 & 0.45 & 46 & 2.68 & 0.36 & 46 & & Not estimable & & \\
\hline Song 2018 & 2.18 & 0.39 & 49 & 2.77 & 0.43 & 49 & 24.2 & $-0.59[-0.75,-0.43]$ & - - & \\
\hline Song and Ding 2012 & 1.63 & 0.67 & 49 & 2.37 & 0.52 & 49 & 11.4 & $-0.74[-0.98,-0.50]$ & -1 & \\
\hline Wu 2017 & 1.31 & 0.9 & 70 & 2.36 & 0.68 & 70 & 9.2 & $-1.05[-1.31,-0.79]$ & & \\
\hline Yuan 2018 & 1.82 & 0.74 & 34 & 2.51 & 0.68 & 35 & 5.7 & $-0.69[-1.03,-0.35]$ & & \\
\hline Zeng 2012 & 1.68 & 0.63 & 42 & 2.38 & 0.56 & 42 & 9.9 & $-0.70[-0.95,-0.45]$ & $\longrightarrow$ & \\
\hline Zhao et al. 2016 & 2.24 & 0.31 & 58 & 2.97 & 0.48 & 57 & 29.2 & $-0.73[-0.88,-0.58]$ & - & \\
\hline Total (95\% CI) & & & 382 & & & 382 & 100.0 & $-0.72[-0.80,-0.64]$ & $\nabla$ & \\
\hline $\begin{array}{l}\text { Heterogeneity: } \mathrm{Chi}^{2}= \\
\text { Test for overall effect: }\end{array}$ & $\begin{array}{l}0, d f=6 \\
17.72(\end{array}$ & $\begin{array}{l}(P=0 \\
<0.0\end{array}$ & $\begin{array}{l}20) ; I^{2} \\
001)\end{array}$ & $=30 \%$ & & & & & $\begin{array}{lll}-1 & -0.5 & 0\end{array}$ & $0.5 \quad 1$ \\
\hline & & & & & & & & & Favours (experimental) & Favours (control) \\
\hline
\end{tabular}

(d)

FIGURE 4: Forest plot of TXLC plus atorvastatin versus atorvastatin in decreasing TC, TG, and LDL-C and in increasing HDL-C: (A) the forest plot of TC, (B) the forest plot of TG, (C) the forest plot of HDL-C, and (D) the forest plot of LDL-C. 
TXLC plus atorvastatin could substantially reduce the level of TG compared with atorvastatin treatment $(\mathrm{MD}=-0.73$; 95\% CI, $-0.81,-0.65 ; P<0.00001$; Figure $4(\mathrm{~b}))$. Due to the limited number of studies, funnel plot analysis was not available.

3.6.4. Meta-Analysis Based on High-Density Lipoprotein Cholesterol (HDL-C). A total of 6 trials with 643 patients evaluated HDL-C and were pooled with a fixed model $[19,24,26,29,31,32]$. The heterogeneity of the HDL-C study was considerably low $\left(P=0.24 ; I^{2}=26 \%\right)$. The results showed that TXLC plus atorvastatin was superior to atorvastatin treatment to increase HDL-C; the difference was statistically significant $(\mathrm{MD}=0.27 ; 95 \% \mathrm{CI}, 0.23,0.31$; $P<0.00001$; Figure $4(\mathrm{c}))$. Due to the limited number of studies, funnel plot analysis was not available.

3.6.5. Meta-Analysis Based on Low-Density Lipoprotein Cholesterol ( $L D L-C)$. Eight trials assessed the therapy of TXLC plus atorvastatin versus atorvastatin in decreasing LDL-C in patients with CHD [19, 21, 24-26, 29, 31, 32]. It has considerably high heterogeneity in LDL-C $(P<0.010$; $\left.I^{2}=62 \%\right)$; after excluding studies that may cause heterogeneity [26], the heterogeneity reduced $\left(P=0.20 ; I^{2}=30 \%\right)$, so a fixed effects model was conducted for analysis. The results showed that TXLC plus atorvastatin was superior to atorvastatin treatment to reduce LDL-C; the difference was statistically significant $(\mathrm{MD}=-0.72 ; 95 \% \mathrm{CI},-0.80,-0.64$; $P<0.00001$; Figure $4(\mathrm{~d}))$. Due to the limited number of studies, funnel plot analysis was not available.

3.6.6. Meta-Analysis Based on C-Reactive Protein (CRP). A total of 7 trials with 690 patients evaluated CRP and were pooled with a random model [18, 23-28]. The heterogeneity was considerably high $\left(P<0.00001 ; I^{2}=85 \%\right)$. Due to the different measurement units used in CRP in various studies, we use SMD as the effect indicator for meta-analysis. The results showed that TXLC plus atorvastatin was superior to atorvastatin treatment to reduce CRP; the difference was statistically significant $(\mathrm{SMD}=-2.06 ; 95 \% \mathrm{CI},-2.56,-1.57$; $P<0.00001$; Table 4). Due to the limited number of studies, funnel plot analysis was not available. Sensitivity analysis found that excluding 7 studies, the combined effects were still statistically significant, and the direction of the forest plot results did not change.

3.6.7. Meta-Analysis Based on Frequency of Angina Pectoris. Five trials with 532 participants assessed the effect of TXLC plus atorvastatin versus atorvastatin in decreasing the frequency of angina pectoris in patients with $\mathrm{CHD}$ and were pooled with a random model [22, 23, 27, 28, 31]. It has considerably high heterogeneity in the frequency of angina pectoris $\left(P<0.00001 ; I^{2}=88 \%\right)$. Due to the different measurement units used for the frequency of angina pectoris in various studies, we use SMD as an effective indicator for meta-analysis. The results showed that TXLC plus atorvastatin was superior to atorvastatin treatment to reduce the frequency of angina pectoris; the difference was statistically significant $(\mathrm{SMD}=-1.41 ; 95 \% \mathrm{CI},-1.97,-0.85 ; P<0.00001$; Table 4). Due to the limited number of studies, funnel plot analysis was not available.

3.6.8. Meta-Analysis Based on Duration of Angina Pectoris. Four trials with 372 participants assessed the effect of TXLC plus atorvastatin versus atorvastatin in decreasing the duration of angina pectoris [22, 23, 27, 30]. There was considerable heterogeneity in the duration of angina pectoris $\left(P<0.00001, I^{2}=93 \%\right)$ in trials. Meta-analysis with a random effects model showed that compared with atorvastatin treatment, TXLC plus atorvastatin significantly improves angina symptoms. The pooled analysis indicated that there was a statistically significant difference between TXLC plus atorvastatin and atorvastatin treatment to reduce the duration of angina pectoris $(\mathrm{MD}=-2.30 ; 95 \% \mathrm{CI},-3.39,-1.21$; $P<0.0001$; Table 4). Due to the limited number of studies, funnel plot analysis was not available. Sensitivity analysis found that excluding 4 studies, the combined effects were still statistically significant, and the direction of the forest plot results did not change.

3.6.9. Meta-Analysis of Adverse Reactions. Six trials with 695 participants assessed the effect of TXLC plus atorvastatin versus atorvastatin in adverse reactions [19-21, 24, 29, 31]. The results showed that there was no heterogeneity $\left(P=0.92 ; I^{2}=0 \%\right)$. Meta-analysis with a fixed effects model showed that compared with atorvastatin treatment, TXLC plus atorvastatin significantly improves angina symptoms. The pooled analysis indicated that there was no significant difference between TXLC plus atorvastatin and atorvastatin treatment on adverse reactions $(\mathrm{RR}=0.84 ; 95 \% \mathrm{CI}, 0.51,1.39 ; P=0.50$; Table 4$)$. Due to the limited number of studies, funnel plot analysis was not available.

3.7. Subgroup Analysis. Through the meta-analysis of CRP and the frequency of angina pectoris, we believe that the reason for the high heterogeneity may be due to the different measurement units used in the two outcome indicators in each study. Therefore, subgroup analysis of CRP and frequency of angina pectoris is performed according to the different measurement units. In the subgroup analysis of CRP, 5 studies $[18,23,25,27,28]$ used " $\mathrm{mg} / \mathrm{L}$ " as the measurement unit, and 2 studies $[24,26]$ used " $\mathrm{mmol} / \mathrm{L}$ " as the measurement unit. The results showed that the subgroup with the measurement unit "mg/L" still has heterogeneity $\left(P<0.00001 ; I^{2}=97 \%\right.$; Figure 5$)$, and the subgroup with the unit of measurement " $\mathrm{mmol} / \mathrm{L}$ " has no heterogeneity $\left(P=0.55 ; I^{2}=0 \%\right.$; Figure 5$)$, indicating that the heterogeneity is affected by the inconsistent measurement unit, but there may be other factors. In the subgroup analysis of the frequency of angina pectoris, 3 studies [22, 27, 28] used "times/day" as the measurement unit, and 2 studies $[23,30]$ used "times/week" as the measurement unit, and the results showed that the subgroup with the measurement unit 
TABLE 4: Meta-analysis results of important outcome measures.

\begin{tabular}{lcccccc}
\hline Outcome measures & Number of included studies & $\begin{array}{c}\text { Results of } \\
\text { heterogeneity } \\
\text { test }\end{array}$ & Effect model & Results of meta-analysis \\
\hline CRP & 7 & $I^{2}(\%)$ & $P$ & & $95 \%$ CI \\
Frequency of angina pectoris & 5 & 85 & $<0.00001$ & Random & SMD $=-2.06(-2.56,-1.57)$ & $<0.00001$ \\
Duration of angina pectoris & 4 & 88 & $<0.00001$ & Random & SMD $=-1.41(-1.97,-0.85)$ & $<0.00001$ \\
Adverse reactions & 6 & 93 & $<0.00001$ & Random & MD $=-2.30(-3.39,-1.21)$ & $<0.0001$ \\
\hline
\end{tabular}

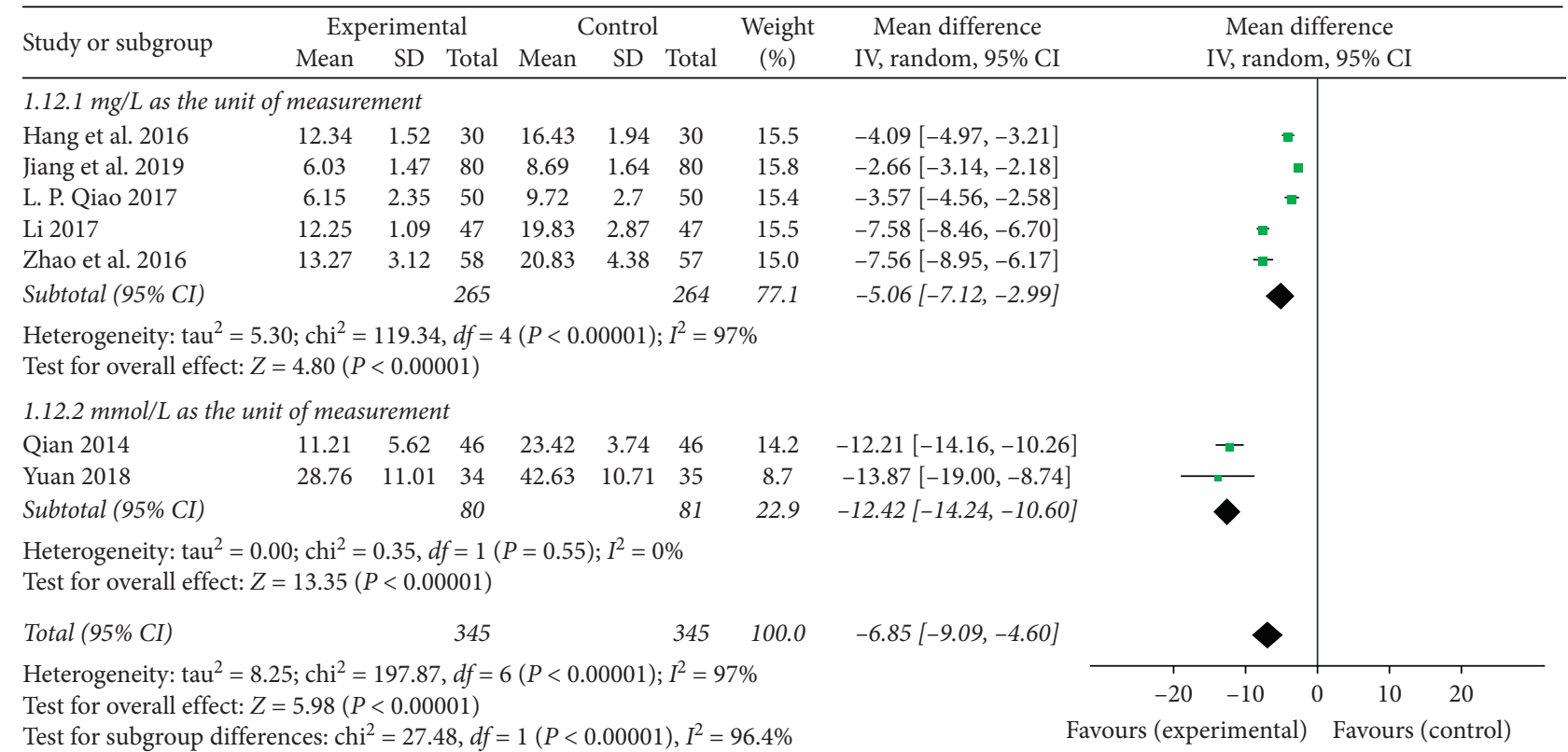

FIGURE 5: Forest plot of subgroup analysis on the CRP of TXLC plus atorvastatin versus atorvastatin in the treatment of CHD.

"times/day" has no heterogeneity $\left(P=0.56 ; I^{2}=0 \%\right.$; Figure 6), and the subgroup with the measurement unit "time/ week" has heterogeneity $\left(P<0.00001 ; I^{2}=98 \%\right.$; Figure 6), indicating that the heterogeneity is affected by the inconsistency of the measurement unit, but there may still be other factors.

3.8. Publication Bias. Publication bias was assessed using a funnel plot based on the effectiveness of clinical treatment reported in 14 studies. The funnel plot was asymmetrical (Figure S1). Furthermore, through Egger's test $P=0.001$ (Figure S2), the results showed that there was publication bias among the studies. And the bias might result from these reasons: small sample size, poor quality, and a high proportion of positive results.

3.9. GRADE Evidence Quality. This study has 9 outcome indicators, which are 5 critical outcome indicators: effectiveness of clinical treatment, TC, TG, HDL-C, LDL-C and 4 important outcome indicators: frequency of angina pectoris, duration of angina pectoris, CRP, and adverse reactions; the GRADE system evidence level of each outcome and the reasons for the promotion and demotion are shown in Table 5.

\section{Discussion}

4.1. Evaluation of Clinical Effectiveness and Safety. CHD belongs to the category of "thoracodynia" in TCM [35]. The occurrence of the disease is related to poor blood circulation in the body, and it leads to stasis of qi and blood and damaged blood vessels and further causes various symptoms such as paroxysmal chest pain [36]. Various factors such as overwork, emotional stress, stress, circulation factors, and so on may cause the onset of this symptom [37], and the sustained development of angina may lead to interruption of blood supply and myocardial infarction. Its main pathogenesis includes coronary artery lipid deposition, atherosclerotic plaque formation, and disorders of lipid metabolism. Therefore, the key to the treatment of CHD is to effectively adjust the blood lipid concentration, improve the tolerance of myocardial cells to ischemia, and improve the blood hypercoagulability state while improving myocardial blood supply [38]. TXLC has the effect of nourishing qi and activating blood, dredge meridians, and pain relief. Basic research proves that TXLC can promote the improvement of 


\begin{tabular}{|c|c|c|c|c|c|c|c|c|c|c|}
\hline \multirow{2}{*}{ Study or subgroup } & \multicolumn{3}{|c|}{ Experimental } & \multicolumn{3}{|c|}{ Control } & \multirow{2}{*}{$\begin{array}{l}\text { Weight } \\
(\%)\end{array}$} & \multirow{2}{*}{$\begin{array}{c}\text { Mean difference } \\
\text { IV, random, 95\% CI }\end{array}$} & \multirow{2}{*}{\multicolumn{2}{|c|}{$\begin{array}{c}\text { Mean difference } \\
\text { IV, random, 95\% CI }\end{array}$}} \\
\hline & Mean & $\mathrm{SD}$ & Total & Mean & SD & Total & & & & \\
\hline \multicolumn{11}{|c|}{ 1.14.1 times/day as the unit of measurement } \\
\hline Jiang et al. 2019 & 1.41 & 0.62 & 80 & 2.64 & 0.77 & 80 & 21.4 & $-1.23[-1.45,-1.01]$ & घ & \\
\hline L. P. Qiao 2017 & 1.4 & 1.3 & 50 & 2.5 & 1.6 & 50 & 19.0 & $-1.10[-1.67,-0.53]$ & - & \\
\hline Nie 2018 & 1.63 & 0.41 & 39 & 2.64 & 1.03 & 39 & 20.7 & $-1.01[-1.36,-0.66]$ & - & \\
\hline Subtotal (95\% CI) & & & 169 & & & 169 & 61.2 & $-1.16[-1.34,-0.99]$ & 1 & \\
\hline \multicolumn{11}{|c|}{$\begin{array}{l}\text { Heterogeneity: } \operatorname{tau}^{2}=0.00 ; \mathrm{chi}^{2}=1.16, d f=2(P=0.56) ; I^{2}=0 \% \\
\text { Test for overall effect: } Z=13.01(P<0.00001)\end{array}$} \\
\hline \multicolumn{11}{|c|}{ 1.14.2 times/week as the unit of measurement } \\
\hline Li 2017 & 5.76 & 1.2 & 47 & 9.31 & 1.65 & 47 & 18.9 & $-3.55[-4.13,-2.97]$ & -- & \\
\hline Wang 2018 & 3.34 & 1.25 & 50 & 4.43 & 1.16 & 50 & 19.9 & $-1.09[-1.56,-0.62]$ & - & \\
\hline Subtotal (95\% CI) & & & 97 & & & 97 & 38.8 & $-2.31[-4.72,0.10]$ & & \\
\hline \multicolumn{11}{|c|}{$\begin{array}{l}\text { Heterogeneity: } \mathrm{tau}^{2}=2.95 ; \mathrm{chi}^{2}=41.24, d f=1(P<0.00001) ; I^{2}=98 \% \\
\text { Test for overall effect: } Z=1.88(P=0.06)\end{array}$} \\
\hline Total (95\% CI) & & & 266 & & & 266 & 100.0 & $-1.57[-2.27,-0.88]$ & & \\
\hline \multicolumn{9}{|c|}{$\begin{array}{l}\text { Heterogeneity: } \operatorname{tau}^{2}=0.57 ; \mathrm{chi}^{2}=61.33, d f=4(P<0.00001) ; I^{2}=93 \% \\
\text { Test for overall effect: } Z=4.44(P<0.00001) \\
\text { Test for subgroup differences: } \mathrm{chi}^{2}=0.87, d f=1(P=0.35), I^{2}=0 \%\end{array}$} & Favours (experimental) & $\begin{array}{c}24 \\
\text { Favours (control) }\end{array}$ \\
\hline
\end{tabular}

FIGURE 6: Forest plot of subgroup analysis on the frequency of angina pectoris of TXLC plus atorvastatin versus atorvastatin in the treatment of $\mathrm{CHD}$

microcirculation, relieve atherosclerosis, and has the effect of inhibiting the inflammatory response and thrombosis [39-41]. The active ingredients in TXLC can promote the reduction of lipid deposition in plaques and have an inhibitory effect on the infiltration of inflammatory cells [42,43], so they can be used for the treatment of cardiovascular and cerebrovascular diseases and relieve angina $[44,45]$. Atorvastatin calcium is a selective and competitive inhibitor of HMG-CoA reductase [46]. The drug has the effects of regulating lipids, inhibiting endogenous cholesterol synthesis, and reducing inflammatory cytokine levels [47-49]. Recent studies showed that atorvastatin combined with TXLC can further reduce blood lipid levels, while the incidence of adverse events did not increase [50]. The combined use of the two not only produced beneficial effects on blood lipids but also effectively relieved myocardial ischemia, inhibited inflammation, and resisted atherosclerosis [51].

This study conducted a systematic evaluation according to the PRISMA guidelines and AMSTAR 2 standards. The results showed that the report of the systematic evaluation was not sufficient, and the methodological quality evaluation of all RCTs was of low or critically low quality, suggesting that the quality of the systematic review needs to be further improved. Through a comprehensive analysis of the outcome indicators of the included 15 studies, the results of the meta-analysis showed that TXLC combined with the atorvastatin group was significantly better than the atorvastatin group in terms of clinical treatment effectiveness. In terms of blood lipid levels, the treatment group can lower the levels of TC, TG, and LDL-C and increase the level of HDL-C better than the control group. In terms of inflammatory factors, the treatment group can reduce the level of CRP more than the control group. However, the heterogeneity among the researches of various indicators is large, suggesting poor stability. For the CRP and frequency of angina pectoris, due to the different measurement units between the studies, the subgroup analysis of the CRP and frequency of angina pectoris based on the measurement unit shows that the heterogeneity is affected by the measurement unit to some extent, but it still exists other influencing factors. A total of 6 studies in the included studies reported adverse events, mainly including gastrointestinal reactions such as nausea, vomiting, loss of appetite, muscle aches, abnormal liver function, and so on. No serious adverse reactions occurred. And the results showed that there was no statistical significance between the two groups, suggesting that the safety effect may not be obvious. At the same time, since most studies did not mention adverse events, it is recommended to increase the importance of drug safety in the future and improve the observation and reporting of safety indicators to increase the clinical reference value of the research.

4.2. Limitations of This Study. The study implemented strict inclusion and exclusion criteria. However, as the meta-analysis is a secondary study, it also has certain limitations, mainly considering factors, limited sample sizes, and changes in treatment time. High heterogeneity still exists among some of the outcome indicators. The following five issues remain in all RCTs from the results: (1) The amount of included trials is small, in addition to the lack of high-quality and large sample study. (2) Quality is generally low; the random application is less; and blind implementation is unknown. (3) Partial outcome indicators are subject to publication bias. Although extensive search strategies are used, supplements such as supplements, conference papers, and some gray literature are not available, and the inclusion of research information is limited. The study can only evaluate relevant indicators and cannot eliminate potential publication bias. (4) Languages, regions, and so on are also an issue. Although the language search is not restricted in this research, 15 articles were included in the Chinese literature and 0 articles in English after 


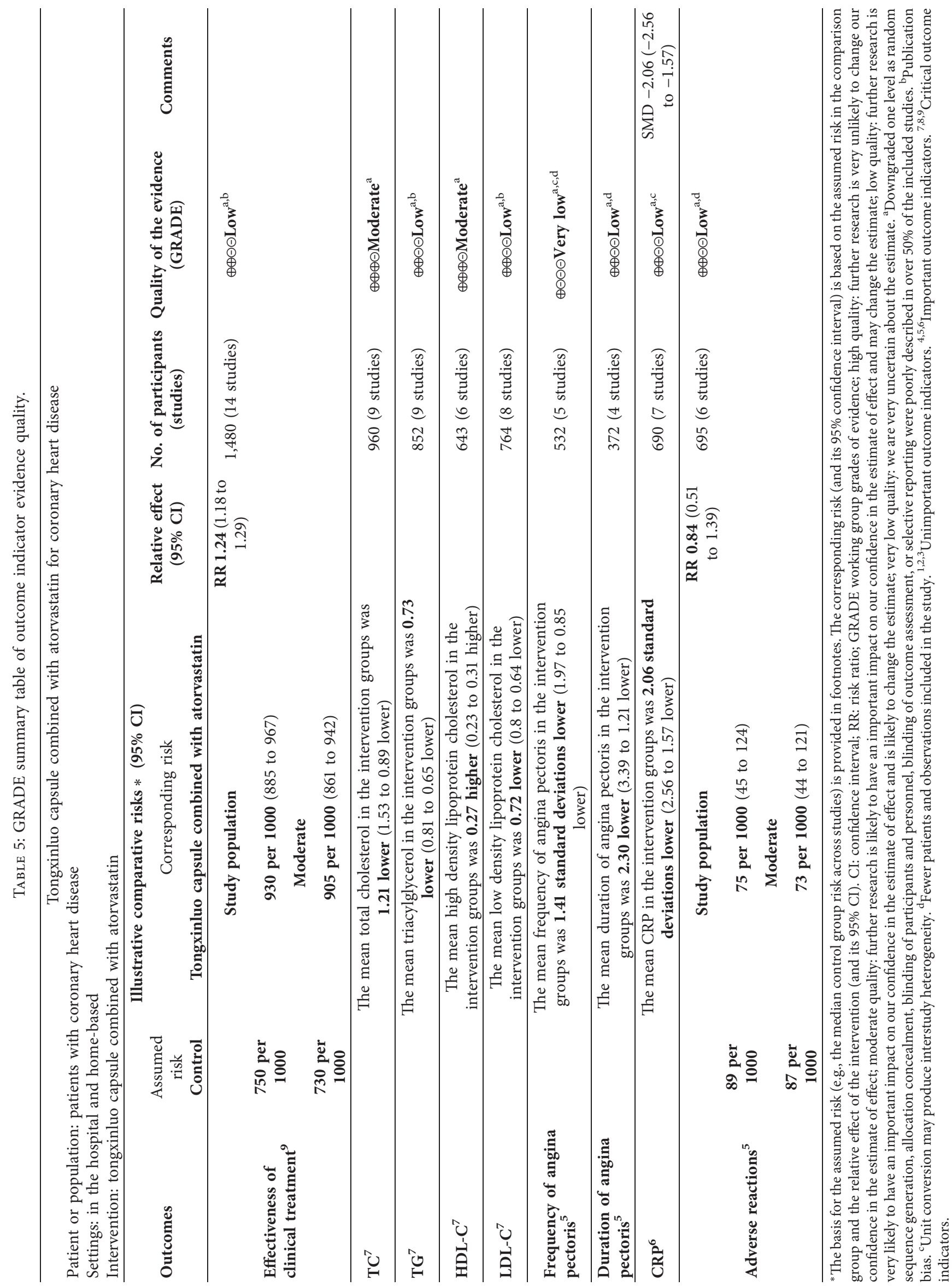


the search, which may affect the extrapolation of the research results. (5) Most of the studies did not report adverse reactions.

4.3. GRADE Systematic Evaluation of Evidence Quality and Recommendation Grade. In the GRADE system, although the evidence based on RCTs was initially rated as high quality, our confidence in this type of evidence may be reduced by five points. According to the GRADE methodology quality evaluation, two outcome indicators were rated as moderate; six outcome indicators were rated as low; and one outcome indicator was rated as very low for the following reasons. (1) Hidden and blind methods are missing. Therefore, there are research limitations. (2) Unit conversion may lead to heterogeneity between studies. (3) Some studies include fewer patients and observations, and the results are inaccurate. (4) There was publication bias. At the same time, the critical outcome indicators of this study are the effectiveness of clinical treatment and blood lipid indicators (TC, TG, LDL$\mathrm{C}$, and HDL-C), and the quality of evidence is generally low. And in view of the low quality of included RCTs, the authenticity of the conclusions was affected. These studies are all domestic and affect the extrapolation of conclusions, so the recommendation level is a weak recommendation.

\section{Conclusions}

In summary, the results of systematic review and meta-analysis suggest that TXLC combined with atorvastatin would benefit patients with CHD. However, based on the GRADE system, the recommendation level is a weak recommendation, and the quality of articles is low; more accurate conclusions may need to be collected more new research data, a full review of other language literature, and so on. At the same time, a more rigorous trial design is indispensable, especially the need for large-scale, multicenter, randomized, randomized, and double-blind RCTs. It is recommended to design large samples and high-quality research and adopt key indicators in strict accordance with the consolidated standards of reporting trials (CONSORT) standard [52] for further demonstrations, so as to draw more reliable conclusions to guide clinical practice.

\section{Abbreviations}

CHD: $\quad$ Coronary heart disease

TXLC: $\quad$ Tongxinluo capsule

TCM: $\quad$ Traditional Chinese medicine

PRISMA: Preferred reporting items for systematic reviews and meta-analyses

AMSTAR Assess the methodological quality of

2: $\quad$ systematic reviews

GRADE: Grading of Recommendations Assessment,

TC: $\quad$ Total cholesterol

TG: $\quad$ Triglycerides

LDL-C: $\quad$ Low-density lipoprotein cholesterol

HDL-C: High-density lipoprotein cholesterol

CRP: C-reactive protein

RCTs: $\quad$ Randomized controlled trials

RR: $\quad$ Risk ratio
CI: $\quad$ Confidence interval

MD: $\quad$ Mean difference

SMD: $\quad$ Standardized mean difference

NR: $\quad$ Not report

tid: $\quad$ Ter in die

CONSORT: Consolidated standards of reporting trials.

\section{Data Availability}

No data were used to support this study.

\section{Disclosure}

Qiao Liu and Taiwei Dong are the co-first authors.

\section{Conflicts of Interest}

The authors declare that they have no conflicts of interest.

\section{Authors' Contributions}

Qiao Liu and Peifeng Wei designed the study. Qiao Liu and Taiwei Dong carried out literature research. Taiwei Dong and Miaomiao Xi acquired data. Qiao Liu and Licheng Gou carried out a statistical analysis. Qiao Liu and Yang Bai edited the manuscript. Lian Hou, Min Li, and Li Ou revised/ reviewed the manuscript. Feng Miao and Peifeng Wei approved the final version of the manuscript. Qiao Liu and Taiwei Dong contributed equally to this work.

\section{Acknowledgments}

This work was financially supported by grants from the Innovative Team Plan of the Second Affiliated Hospital of Shaanxi University of Chinese Medicine (2020XKTD-A04) and the Subject Innovation Team of Shaanxi University of Chinese Medicine (2019-QN02).

\section{Supplementary Materials}

Supplementary data to this article can be found in supplementary materials. Figure S1: funnel plot of the effectiveness of clinical treatment. Figure S2: Egger's test. (Supplementary Materials)

\section{References}

[1] P. Ong, A. Aziz, H. S. Hansen et al., "Structural and functional coronary artery abnormalities in patients with vasospastic angina pectoris," Circulation Journal, vol. 79, no. 7, pp. 1431-1438, 2015.

[2] R. A. H. Stewart, D. M. Colquhoun, S. L. Marschner et al., "Persistent psychological distress and mortality in patients with stable coronary artery disease," Heart, vol. 103, no. 23, pp. 1860-1866, 2017.

[3] H. Z. Chen and G. W. Lin, Practical Internal Medicine, People's Medical Publishing House, Beijing, China, 2009.

[4] R. Li, Y. Zhen, and Y. P. Xiao, "Evaluation of the therapeutic effect and clinical safety of atorvastatin calcium combined with ezetimibe on coronary heart disease," Journal of 
Cardiopulmonary and Vascular Disease, vol. 35, no. 4, pp. 266-268, 2016.

[5] W. E. Stehbens, "Coronary heart disease, hypercholesterolemia, and atherosclerosis II. Misrepresented data," Experimental and Molecular Pathology, vol. 70, no. 2, pp. 120-139, 2001.

[6] T. F. Whayne Jr, "Assessment of low-density lipoprotein targets," Angiology, vol. 64, pp. 411-416, 2013.

[7] T. T. Liu, "Explore the effect and clinical safety of atorvastatin calcium combined with new ezetimibe drugs in the treatment of coronary heart disease," Chinese Medicine Guide, vol. 26, no. 23, pp. 113-114, 2018

[8] Y. Y. Zhang, "Analysis of therapeutic effect of Xintong oral liquid on unstable Angina pectoris of coronary heart disease," Modern Journal of Integrated Traditional Chinese and Western Medicine, vol. 24, no. 31, pp. 3473-3475, 2015.

[9] R. Puri, S. E. Nissen, P. Libby et al., "C-reactive protein, but not low-density lipoprotein cholesterol levels, associate with coronary atheroma regression and cardiovascular events after maximally intensive statin therapy," Circulation, vol. 128, no. 22, pp. 2395-2403.

[10] M. Uematsu, T. Nakamura, W. Suqamata et al., "Echolucency of carotid plaque is useful for assessment of residual cardiovascular risk in patients with chronic coronary artery disease who achieve LDL-C goals on statin therapy," Circ J, vol. 78, no. 1, pp. 151-158, 2013.

[11] H. Xu, X. Y. Lu, K. Y. Chen et al., "Study on the correlation of blood stasis syndrome and its concurrent syndrome with coronary angiography and restenosis after intervention," Chinese Journal of Integrated Traditional Chinese and Western Medicine, vol. 27, no. 1, pp. 8-13, 2007.

[12] L. Zhang, D. W. Zhang, J. G. Liu et al., "Protective effects of Chinese medicine compatibility of promoting blood circulation and detoxification on adapted myocardial tissues of rats," Chinese Journal of Integrated Traditional Chinese and Western Medicine Emergency, vol. 17, no. 3, pp. 134-137, 2010.

[13] Y. H. Sun, "Study on the value of Tongxinluo capsule in the clinical treatment of coronary heart disease and angina pectoris," Integrated Traditional Chinese and Western Medicine Cardiovascular Disease, vol. 6, no. 21, p. 68, 2018.

[14] X. S. Huang, Q. D. Yang, G. Y. Long et al., "Clinical study on the effect of Tongxinluo capsule on unstable plaque of cervical atherosclerosis," Journal of Intractable Diseases, vol. 6, no. 5, pp. 267-269, 2007.

[15] T. Dong, J. Wang, X. Ma et al., "Shexiang baoxin pills as an adjuvant treatment for chronic heart failure: a system review and meta-analysis," Evidence-Based Complementary and Alternative, vol. 2018, Article ID 6949348, 2018.

[16] D. Moher, A. Liberati, J. Tetzlaff et al., "Preferred reporting items for systematic reviews and meta-analyses: the PRISMA statement," Journal of Clinical Epidemiology, vol. 62, pp. 1006-1012, 2009.

[17] B. J. Shea, B. C. Reeves, G. Wells et al., "AMSTAR 2: a critical appraisal tool for systematic reviews that include randomised or non-randomised studies of healthcare interventions, or both," BMJ, vol. 358, p. j4008, 2017.

[18] L. Hang, J. H. Tian, and J. Li, "Effect of atorvastatin combined with Tongxinluo capsule on cTnI, HbA1c and clinical efficacy in patients with coronary heart disease," Journal of Cardiovascular and Cerebral Vascular Diseases, vol. 14, no. 15, pp. 1765-1767, 2016.

[19] S. G. Liu and J. An, "Clinical observation of atorvastatin combined with Tongxinluo capsule in the treatment of unstable angina pectoris due to coronary heart disease," Journal of Clinical Rational Use, vol. 9, no. 5, pp. 3-4, 2016.

[20] Y. L. Qiao, "Clinical efficacy and safety analysis of atorvastatin combined with Tongxinluo capsule in the treatment of coronary heart disease," Modern Diagnosis and Treatment, vol. 28, no. 11, pp. 2003-2004, 2017.

[21] L. Z. Song, "Effectiveness and safety of Tongxinluo capsule combined with atorvastatin on angina pectoris of coronary heart disease," Electronic Journal of Integrated Traditional Chinese and Western Medicine for Cardiovascular Diseases, vol. 6 , no. 17, p. $49+52,2018$.

[22] F. P. Nie, "Effect of Tongxinluo capsule and atorvastatin calcium on patients with coronary heart disease and unstable angina pectoris," Journal of Contemporary Medicine, vol. 16, no. 7, pp. 107-108, 2018.

[23] H. X. Li, "Effect of Tongxinluo capsule combined with atorvastatin calcium on coronary heart disease unstable angina pectoris," Henan Medical Research, vol. 26, no. 23, pp. 4263-4265, 2017.

[24] L. B. Yuan, "Clinical effect analysis of Tongxinluo capsule combined with atorvastatin in the treatment of coronary heart disease and angina pectoris," Clinical Research, vol. 26, no. 4, pp. 58-60, 2018.

[25] S. Y. Zhao, Z. S. Huang, J. Wen et al., "Clinical observation of Tongxinluo capsule combined with atorvastatin in the treatment of coronary heart disease and angina pectoris," Journal of Practical Cardiocerebral Pulmonary Vascular Disease, vol. 24, no. 8, pp. 96-99, 2016.

[26] J. Qian, "Clinical effect of Tongxinluo combined with atorvastatin on unstable angina pectoris due to coronary heart disease and its effect on high-sensitivity C-reactive protein," Chinese and Foreign Medical Research, vol. 12, no. 35, pp. 19-20, 2014.

[27] L. P. Qiao, "Effect of Tongxinluo combined with atorvastatin on coronary heart disease unstable angina pectoris and its effect on high-sensitivity C-reactive protein," Northern Medicine, vol. 14, no. 8, pp. 141-142, 2017.

[28] H. Y. Jiang, F. Li, and X. S. Zhao, "Effect of Tongxinluo combined with atorvastatin on coronary heart disease unstable angina pectoris and its effect on high-sensitivity C-reactive protein," China Modern Drug Application, vol. 13, no. 17, pp. 57-59, 2019.

[29] J. L. Wu, "Clinical effect of Tongxinluo combined with atorvastatin on unstable angina pectoris due to coronary heart disease," Chinese Contemporary Medicine, vol. 25, no. 17, pp. 124-126, 2017.

[30] E. Z. Wang, "Effect of Tongxinluo and Atorvastatin on unstable angina pectoris due to coronary heart disease," Smart Health, vol. 4, no. 2, pp. 98-99, 2018.

[31] Y. M. Song and Y. Q. Ding, "Clinical effect of atorvastatin and Tongxinluo capsules on coronary heart disease," Clinical Research in Traditional Chinese Medicine, vol. 4, no. 6, pp. 59-60, 2012.

[32] X. H. Zeng, "Efficacy of atorvastatin combined with Tongxinluo capsule in the treatment of coronary heart disease," China Practical Medicine, vol. 7, no. 16, pp. 171-172, 2012.

[33] D. Atkins, D. Best, P. A. Briss et al., "Grading quality of evidence and strength of recommendations," BMJ, vol. 328, no. 328, p. 1490, 2004.

[34] G. W. Group, "Grade guidelines: 1. Introduction-grade evidence summary table and result summary table," Journal of Clinical Epidemiology, vol. 64, no. 4, pp. 383-394, 2011.

[35] M. H. Sun, Y. Zhang, Y. Xiao et al., "Relationship between different types of coronary heart disease and TCM 
pathogenesis," Journal of Integrated Traditional Chinese and Western Medicine Cardio-Cerebrovascular Disease, vol. 15, no. 4, pp. 507-508, 2017.

[36] J. Y. Wu, L. Zhang, Y. Z. Xie et al., "Study on the mechanism of wendan decoction in treating coronary heart disease based on internet pharmacology," Chinese Pharmacy, vol. 29, no. 23, pp. 3227-3232.

[37] S. Möbius-Winkler, M. Uhlemann, V. Adams et al., "Coronary collateral growth induced by physical exercise: results of the leipzig exercise training versus MEdica management in patients with stable coronary artery (EXCITE) trial," Circulation, vol. 133, no. 15, 2016.

[38] V. V. Kalyuzhin, A. T. Teplyakov, E. Y. Pushnikova et al., "Comparative evaluation of the impact of four-week therapy with amlodipine and atenolol on quality of life andblood lipid composition in patients with coronary heart disease associated with metabolic syndrome," Ter Arkh, vol. 85, no. 5, pp. 68-72, 2013.

[39] B. R. Zhang, "Effect of Tongxinluo capsule on angiogenesis function of myocardial ischemia rats," Journal of Taishan Medical College, vol. 36, no. 6, pp. 636-639, 2015.

[40] J. Y. Luo and X. L. Luo, "Tongxinluo capsule for acute coronary syndrome and its effect on high sensitive C-reactive protein," Inner Mongolia Chinese Medicine, vol. 34, no. 8, p. 29, 2015.

[41] S. Zhao, "Clinical observation of Tongxinluo capsule in treating diabetic cardiomyopathy," New World of Diabetes, vol. 35, no. 13, pp. 31-32, 2015.

[42] K. Qi, L. P. Jiang, X. D. Li et al., “Tongxinluo's alleviation of diabetic myocardial ischemia-reperfusion injury is related to its improvement of endothelial barrier function," China Circulation Magazine, vol. 29, no. 12, pp. 1020-1023, 2014.

[43] Q. X. Xue, Y. X. Hou, Y. H. Wang et al., "Analysis of the effect of rosuvastatin combined with Tongxinluo on inflammatory factors and carotid atherosclerosis," Modern Instruments and Medical, vol. 21, no. 1, pp. 93-94, 2015.

[44] H. L. Jia, K. Q. Zhang, and H. T. Liu, "Effects of Tongxinluo combined with trimetazidine on high-sensitivity C-reactive protein and blood lipids in patients with angina pectoris after PCI surgery," Journal of Practical Cardiocerebral Pulmonary Vascular Disease, vol. 20, no. 8, pp. 1288-1290, 2012.

[45] L. Sun, "Clinical observation of Tongxinluo and atorvastatin in patients with unstable angina pectoris," Chinese Journal of Cardiovascular Diseases, vol. 3, no. 35, pp. 5-6, 2015.

[46] A. Katsargyris, C. Klonaris, S. Tsiodras et al., "Statin treatment is associated with reduced toll-like receptor 4 immunohistochemical expression on carotid atherosclerotic plaques: a novel effect of statins," Vascular, vol. 19, no. 6, pp. 320-326, 2011.

[47] L. Yang, "Clinical observation on Tongxinluo capsule combined with atorvastatin in treating 36 cases of angina pectoris due to coronary heart disease," Internal Medicine, vol. 9, no. 6, pp. 641-643, 2014.

[48] Q. Zhou and J. K. Liao, "Statins and cardiovascular diseases: from cholesterol lowering to pleiotropy," Cua Pharm Des, vol. 15, no. 5, pp. 467-478, 2009.

[49] Y. B. Zhou, Y. Gao, J. Sun et al., "Progress in treatment of unstable angina pectoris of coronary heart disease with traditional Chinese medicine," Henan Chinese Medicine, vol. 34, no. 2, pp. 242-243, 2014.

[50] J. J. Li, Clinical Study of Atorvastatin Calcium Combined with Tongxinluo Capsule on Patients with Unstable Angina Pectoris, Yan'an University, Yan'an, China, 2014.
[51] Y. X. Gao, "The efficacy of Tongxinluo combined with atorvastatin in the treatment of unstable angina pectoris of coronary heart disease and its influence on high-sensitivity C-reactive protein," Traditional Chinese Medicine Information, vol. 31, no. 3, pp. 139-141, 2014.

[52] K. F. Schulz, D. Moher, and D. G. Altman, "CONSORT 2010 comments," Lancet, vol. 376, no. 9748, pp. 1222-1223, 2010. 